\title{
Lasing action in strongly coupled plasmonic nanocavity arrays
}

\author{
Wei Zhou ${ }^{1 \dagger}$, Montacer Dridi ${ }^{2}$, Jae Yong Suh ${ }^{2}$, Chul Hoon Kim ${ }^{2,3 \dagger}$, Dick T. $\mathrm{Co}^{2,3}$, \\ Michael R. Wasielewski2,3, George C. Schatz ${ }^{2}$ and Teri W. Odom ${ }^{1,2,3 \star}$
}

Periodic dielectric structures are typically integrated with a planar waveguide to create photonic band-edge modes for feedback in one-dimensional distributed feedback lasers and two-dimensional photonic-crystal lasers ${ }^{1-4}$. Although photonic band-edge lasers are widely used in optics and biological applications, drawbacks include low modulation speeds and diffraction-limited mode confinement ${ }^{5,6}$. In contrast, plasmonic nanolasers can support ultrafast dynamics and ultrasmall mode volumes ${ }^{7-9}$. However, because of the large momentum mismatch between their nanolocalized lasing fields and freespace light, they suffer from large radiative losses and lack beam directionality. Here, we report lasing action from bandedge lattice plasmons in arrays of plasmonic nanocavities in a homogeneous dielectric environment. We find that optically pumped, two-dimensional arrays of plasmonic Au or Ag nanoparticles surrounded by an organic gain medium show directional beam emission (divergence angle $<1.5^{\circ}$ and linewidth $<1.3 \mathrm{~nm}$ ) characteristic of lasing action in the far-field, and behave as arrays of nanoscale light sources in the near-field. Using a semi-quantum electromagnetic approach to simulate the active optical responses, we show that lasing is achieved through stimulated energy transfer from the gain to the band-edge lattice plasmons in the deep subwavelength vicinity of the individual nanoparticles. Using femtosecond-transient absorption spectroscopy, we verified that lattice plasmons in plasmonic nanoparticle arrays could reach a 200 -fold enhancement of the spontaneous emission rate of the dye because of their large local density of optical states.

According to Fermi's golden rule ${ }^{10}$, the spontaneous emission rate of an emitter is proportional to the local density of optical states (LDOS) and can be enhanced in an optical microcavity by the Purcell factor ${ }^{11}$

$$
F=\frac{3}{4 \pi^{2}}\left(\frac{Q}{V_{\text {mode }}}\right)\left(\frac{\lambda}{2 n}\right)^{3}
$$

where $Q$ is the cavity quality factor, $V_{\text {mode }}$ is the mode volume, $\lambda$ is the resonant wavelength and $n$ is the refractive index of the medium. A large LDOS can increase not only the rate of spontaneous emission, but also stimulated emission processes in the lasing action, which can improve laser modulation speeds without requiring stronger pump powers ${ }^{5,6,12}$. Plasmonic nanolasers have cavities with ultrasmall $V_{\text {mode }}$, LDOS that can be increased up to 1,000 times, together with femtosecond cavity lifetimes 9 . However, due to losses, the $Q$ for isolated plasmonic cavities is $l_{0}{ }^{13}$. Arrays of plasmonic nanoparticles exhibit suppressed radiative losses because of electromagnetic interactions between individual units $^{14-20}$. These delocalized lattice plasmons can be treated as Bloch modes, which are inherent to two-dimensional periodic metal nanostructures ${ }^{18,20}$ and show spatial dispersion and form standing waves with a low group velocity $\left(v_{\mathrm{g}}=\delta \omega / \delta k_{\|}\right)$at their band-edge states. Unlike Bloch modes in two-dimensional photonic-crystal slabs, which require a multilayer waveguide structure to achieve vertical confinement by total internal reflection ${ }^{21,22}$, lattice plasmons can exist within a uniform dielectric environment ${ }^{15,18,19}$ and also sustain a large LDOS, because their intense optical fields are coupled to oscillating charges in the metal nanostructures ${ }^{16,19,20,23}$.

Our plasmonic nanocavity array laser is illustrated schematically in Fig. 1a. Briefly, two-dimensional arrays of Au nanoparticles were patterned on a glass substrate and then covered by a polymer gain layer composed of polyurethane and IR-140 dye (see Methods). Angle-resolved extinction measurements revealed that the lattice plasmons supported in the two-dimensional $\mathrm{Au}$ nanoparticle arrays were dispersive. Notably, the resonant wavelengths of the lattice plasmons were blueshifted from $913 \mathrm{~nm}$ to $850 \mathrm{~nm}$ and followed the $(0, \pm 1)$ Rayleigh anomaly line in polyurethane as the incident angle $\theta$ increased from $0^{\circ}$ to $40^{\circ}$ (Fig. 1b). Displaying the measured extinction spectra in the form of a dispersion diagram revealed that lattice plasmons evolved from a band-edge mode with a low (near-zero) $v_{\mathrm{g}}$ to a propagating mode with larger $v_{\mathrm{g}}$ (Supplementary Fig. S1). Figure 1c presents the normal incidence extinction spectra of a Au nanoparticle array with and without the gain layer. Here, the broad peak at $820 \mathrm{~nm}$ corresponds to the absorption of the IR-140 dye, and the narrow peak at $913 \mathrm{~nm}$ can be attributed to band-edge lattice plasmons. Numerical calculations (Lumerical FDTD Solutions) of the angle-resolved extinction spectra (Supplementary Fig. S2) were in excellent agreement with the measurements and revealed that the extinction of band-edge lattice plasmons had nearly equal contributions from scattering and absorption losses (Fig. 1d). The calculations also showed that band-edge lattice plasmons induced in-phase oscillations of the dipole moments of individual nanoparticles and formed standing waves in the plane of the nanoparticle arrays with intense optical fields concentrated on the edges of individual $\mathrm{Au}$ nanoparticles (Supplementary Fig. S3). Compared with band-edge states, propagating lattice plasmons had a well-defined phase-front perpendicular to the propagation direction and also larger scattering loss (Supplementary Fig. S4).

Arrays of plasmonic nanoparticles surrounded by gain media were optically pumped using a 800 -nm femtosecond-pulsed laser,

\footnotetext{
'Department of Materials Science and Engineering, Northwestern University, Evanston, Illinois 60208, USA, ${ }^{2}$ Department of Chemistry, Northwestern University, Evanston, Illinois 60208, USA, ${ }^{3}$ Argonne-Northwestern Solar Energy Research (ANSER) Center, Northwestern University, Evanston, Illinois 60208, USA; 'Present address: Department of Chemistry and Chemical Biology, Harvard University, Cambridge, Massachusetts 02138, USA (W.Z.), Max Planck Center for Attosecond Science, POSTECH, Pohang 790-784, South Korea (C.H.K.). *e-mail: todom@northwestern.edu
} 
a
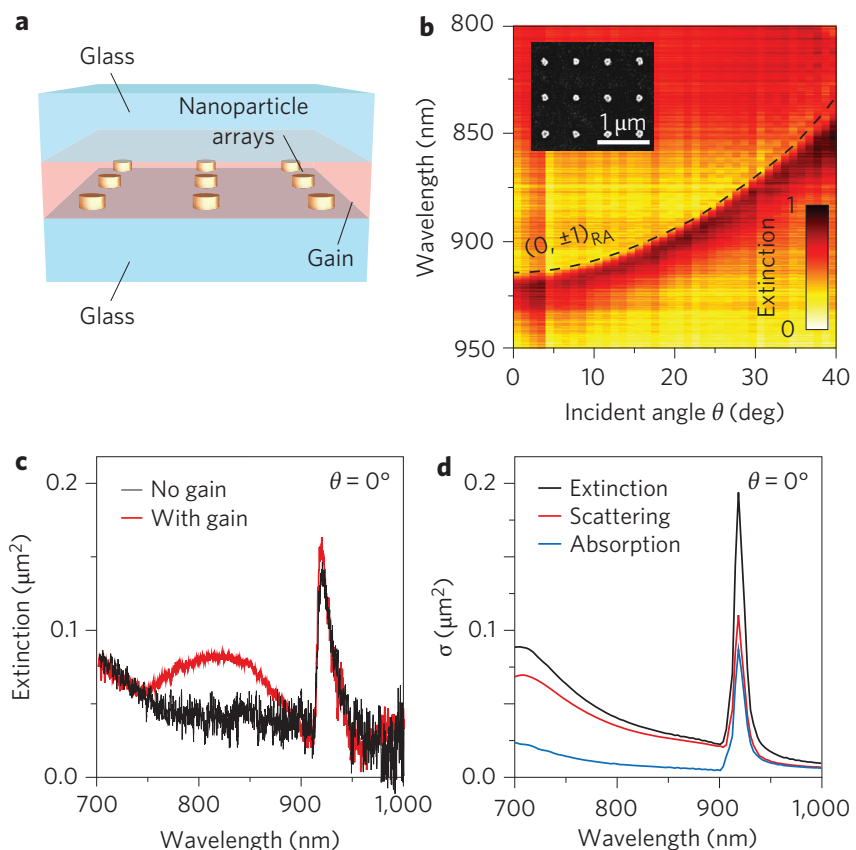

Figure 1 | Plasmonic nanocavity array laser. a, Scheme of the nanoparticle array laser. $\mathbf{b}$, Measured extinction spectra as a function of incident angle $\theta$ (from $0^{\circ}$ to $40^{\circ}$ ) for two-dimensional Au nanoparticle arrays under transverse-magnetic (TM) polarized light. Inset: scanning electron microscopy (SEM) image of Au nanoparticle arrays ( $d=130 \mathrm{~nm}$, $h=50 \mathrm{~nm}, a_{0}=600 \mathrm{~nm}$ ) on a glass substrate. c, Measured spectra of normal incidence extinction of two-dimensional Au nanoparticle arrays without (black curve) and with (red curve) gain. d, Finite-difference time domain (FDTD)-calculated extinction, scattering and absorption spectra of two-dimensional Au nanoparticle arrays under normal incident light.

and the emission spectra were collected at a detection angle of $\alpha=0^{\circ}$ (see Methods and Supplementary Fig. S5). At low pump pulse energies $\left(<0.23 \mathrm{~mJ} \mathrm{~cm}^{-2}\right)$, the emission showed broad spontaneous emission similar to that of the IR-140 gain layer. Above a critical

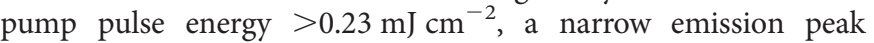
emerged at $\lambda_{\text {em }}=913 \mathrm{~nm}$ with a full-width at half-maximum (FWHM) of $1.3 \mathrm{~nm}$, and the pump-emission (input-output) curves exhibited a threshold behaviour with a dramatic change in slope (Fig. 2a). Defining characteristics of lasers include their high degree of temporal and spatial coherence in their emission ${ }^{24,25}$. Although one of the best indications of temporal coherence is the second-order correlation function $g^{(2)}(t, \tau)$ (ref. 25), this measurement was not possible for plasmonic nanocavity array lasers with organic gain because (i) pulsed pumping at low repetition rates $(10 \mathrm{~Hz}$ to $10 \mathrm{kHz})$ is required to avoid the accumulation of triplet states for organic dyes ${ }^{26}$; (ii) the time resolution of avalanche photodiodes typically used in $g^{(2)}(t, \tau)$ measurements is not enough to resolve non-stationary, pulsed signals with ultrafast decay dynamics ( $\ll 100 \mathrm{ps})$, the timescale inherent to plasmonic nanolasers ${ }^{9}$; and (iii) the relatively poor photostablity of organic dyes in the lasing regime does not allow for a statistically accurate evaluation of temporal coherence in the measurement time.

However, we were able to determine the spatial coherence by mapping the far-field pattern of emitted light using a chargecoupled device (CCD) beam profiler. Once threshold was reached, directional beam emission with a small divergence angle $\left(<1.5^{\circ}\right)$ was observed normal to the sample surface (Fig. 2b,c), so the emitted light was spatially coherent over a large area $\left(>50 \times 50 \mu \mathrm{m}^{2}\right)$ in the nanoparticle arrays. In the quasistatic approximation, a subwavelength plasmonic nanoparticle oscillates like a dipole emitter and radiates spherical waves into the far-field. When arranged in a periodic
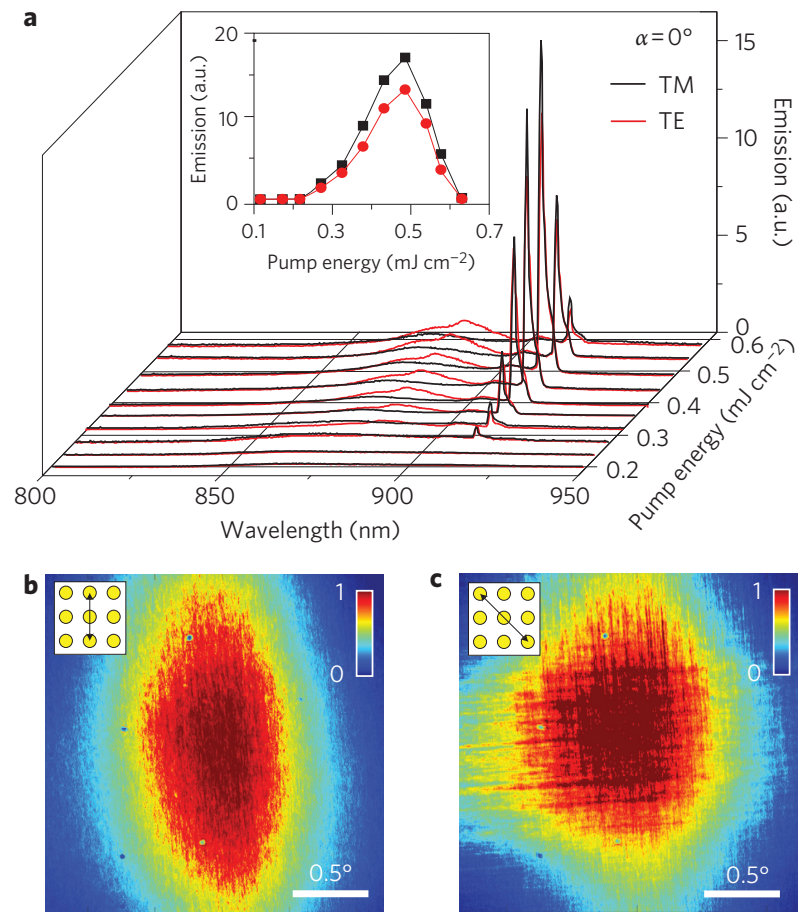

Figure 2 | Coherent lasing action in strongly coupled plasmonic nanoparticle arrays. a, Emission spectra collected at normal detection angle ( $\alpha=0^{\circ}$ ) for different input pump pulse energies and polarizations. Pulsed 800-nm laser light was used to pump two-dimensional Au nanoparticle arrays with gain material at an incident angle of $45^{\circ}$. Inset: output emission intensity as a function of input pump pulse energy. b,c, Far-field pattern of lasing emission beam for pump polarizations parallel (b) and $45^{\circ}$ (c) to the lattice direction of Au nanoparticle arrays.

array, nanoparticles exhibit strong dipole-dipole coupling that can cause in-phase oscillations of resonators (Supplementary Fig. S3d), which results in directional beam emission from constructive interference of the spherical waves. In short, the threshold behaviour in combination with the narrow emission linewidth as well as small beam divergence demonstrates that coherent lasing action can occur in strongly coupled plasmonic nanocavity arrays.

As the pump pulse energies increased further $\left(>0.5 \mathrm{~mJ} \mathrm{~cm}{ }^{-2}\right)$, the intensity of surface-normal lasing at $913 \mathrm{~nm}$ began to saturate and then decreased (Fig. 2a) as new emission features (FWHM $\sim 10 \mathrm{~nm}$ ) from off-normal angles emerged beyond the broad spontaneous emission profile near $870 \mathrm{~nm}$ (Supplementary Figs S6 and S7). Because the IR-140 dye has a broad gain profile (linewidth, $\sim 55 \mathrm{~nm}$ ), strong spectral inhomogeneity can exist in the system as the wavelength of lattice plasmons evolves continuously from $913 \mathrm{~nm}$ at band-edge states to $860 \mathrm{~nm}$ at propagating states. Only molecules emitting near $913 \mathrm{~nm}$ participate efficiently in band-edge lasing action. In contrast, population inversion of molecules emitted near $870 \mathrm{~nm}$ will keep increasing with pump intensity instead of being depleted by band-edge lasing action. Once the optical gain near $870 \mathrm{~nm}$ became high enough with increased pump powers, propagating lattice plasmons were also amplified by stimulated emission as they travelled through the gain medium. Hence, amplified spontaneous emission could be seen at off-normal angles determined by the dispersion relation of the lattice plasmons (Supplementary Fig. S7). Moreover, we found that transverse-electric (TE) polarized pump light cannot excite off-angle amplified spontaneous emission (Supplementary Fig. S6), resulting in higher optical gain and a larger spontaneous emission peak at $870 \mathrm{~nm}$ (Fig. 2a).

To understand the microscopic details of lasing action in plasmonic nanoparticle arrays, we simulated the interaction between the electromagnetic fields and the active gain medium using a 


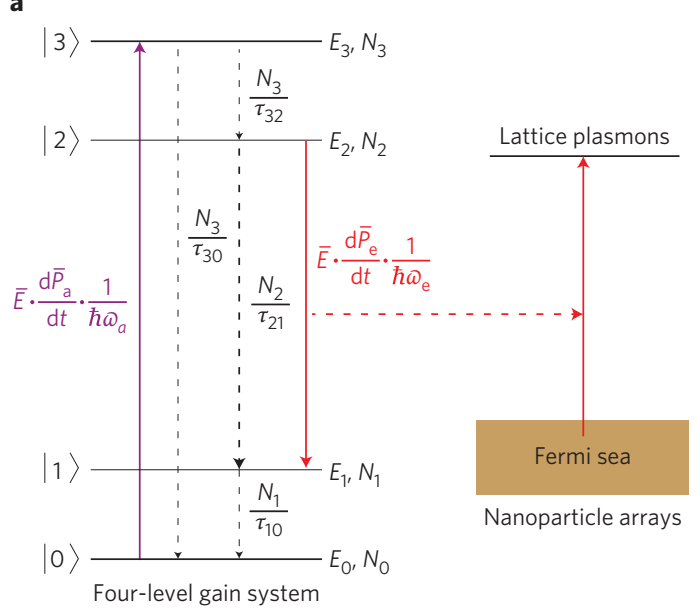

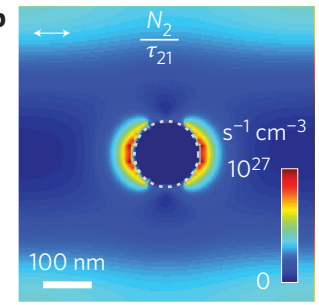

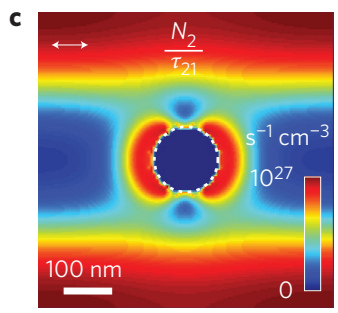

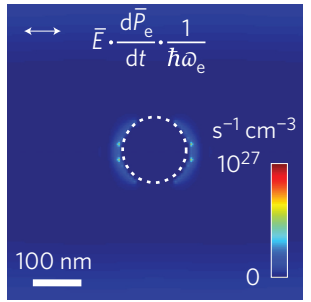
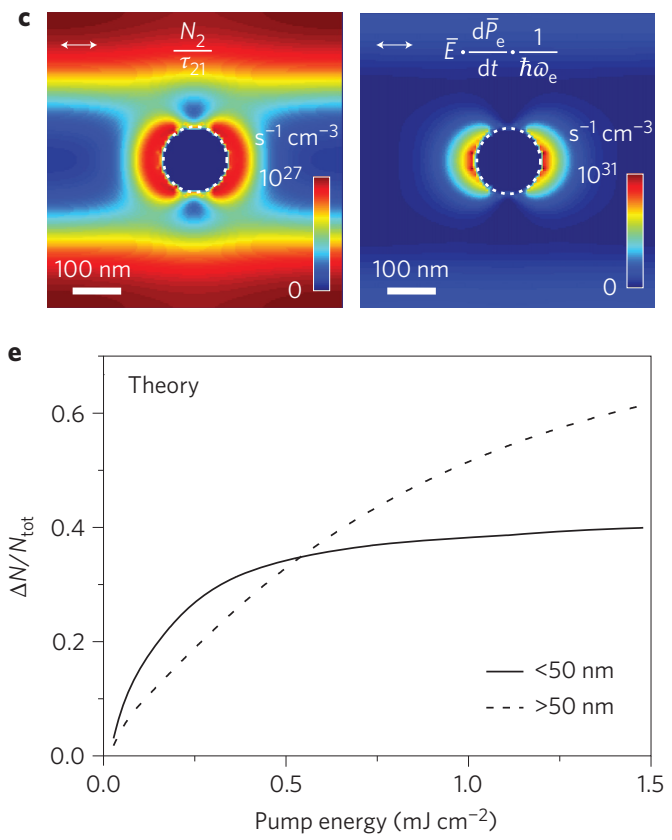

Figure 3 | Nanoscale energy transfer responsible for lasing action. a, Scheme of the energy transfer process from optically excited four-level gain media to lattice plasmons in nanoparticle arrays, where $\bar{E}$ is the total electric field, $\omega_{\mathrm{a}}$ and $\omega_{\mathrm{e}}$ are the absorption and emission frequencies, respectively, and $\bar{P}_{\mathrm{a}}$ and $\bar{P}_{\mathrm{e}}$ are the net macroscopic polarizations resulting from absorption and emission transitions, respectively. b,c, Calculated distribution maps of spontaneous transition rate $N_{2} / \tau_{21}$ and stimulated transition rate (equation (1)) in the middle plane of nanoparticle arrays below (b) and above (c) threshold. $\mathbf{d}$, Calculated emission intensity as a function of input pump pulse energy with different gain volumes surrounding the nanoparticles (at distances of $25 \mathrm{~nm}, 50 \mathrm{~nm}$ and $100 \mathrm{~nm}$ from the nanoparticle surface in all directions, or a uniform $200-\mathrm{nm}$-thick slab). e, Calculated average population inversion density $\Delta \mathrm{N} / \mathrm{N}_{\text {tot }}$ as a function of input pump pulse energy for the gain medium within a distance of $50 \mathrm{~nm}$ from the nanoparticle surface (solid curve), or for the gain medium in a slab excluding the volume within a distance of $50 \mathrm{~nm}$ of the nanoparticle surface (dashed curve).

semi-quantum framework (see Methods and Supplementary Information, page 9) based on a four-level gain system and a timedomain approach (Fig. 3a, Supplementary Fig. S8). As well as reproducing the lasing emission spectra and threshold behaviour in the measurements (Supplementary Fig. S9), the calculations revealed the microscopic energy conversion process in the lasing action (Fig. 3). Below threshold (Fig. 3b), the stimulated transition rate

$$
\frac{1}{\hbar \omega_{\mathrm{e}}} \cdot \bar{E} \cdot \frac{\mathrm{d} \bar{P}_{\mathrm{e}}}{\mathrm{d} t}
$$

from level $|2\rangle$ to level $|1\rangle$ was negligible compared with the spontaneous transition rate $N_{2} / \tau_{21}$. Above threshold (Fig. 3c), the stimulated transition rate was several orders of magnitude higher than the spontaneous transition rate. Significantly, the spatial profile of the stimulated transition rate had maximum values on the edges of the nanoparticles, which was in excellent agreement with the electromagnetic mode profile of band-edge lattice plasmons in the passive system without gain (Supplementary Fig. S3). Thus, lasing action is achieved because the nanolocalized electromagnetic fields of band-edge lattice plasmons stimulate excited-state molecules to transfer energy into plasmons of the same frequency, phase and polarization. Our calculations confirm that only the population inversion in the subwavelength vicinity of the nanoparticles can effectively participate in the lasing action (Fig. 3d) and will be clamped at the lasing threshold (Fig. 3e).

A side-by-side comparison of optical responses between plasmonic and non-plasmonic nanoparticle arrays further supports the critical role of lattice plasmons in the lasing feedback (Fig. 4). Unlike nanoparticles made of non-plasmonic materials $\left(\mathrm{TiO}_{2}\right.$ and $\mathrm{Ti}$ ), nanoparticles made of plasmonic materials ( $\mathrm{Au}$ and $\mathrm{Ag}$ ) have a negative permittivity $\varepsilon_{\mathrm{r} 1}(\omega)$ that enables a large polarizability and a high dipole moment strength $|\mathbf{p}|$ at plasmon resonances ${ }^{13}$. Thus, they can sustain delocalized lattice plasmons because of strong dipole-dipole electromagnetic interactions in periodic arrays $^{14,15}$. Not unexpectedly, both measurements and calculations showed much stronger extinction peaks for $\mathrm{Au}$ and $\mathrm{Ag}$ nanoparticle arrays than for $\mathrm{Ti}$ and $\mathrm{TiO}_{2}$ nanoparticle arrays with the same nanoparticle geometry and lattice periodicity (Supplementary Fig. S10). Moreover, we observed that $\mathrm{Au}$ and $\mathrm{Ag}$ nanoparticle arrays induced narrow and directional lasing emission with threshold behaviours, whereas $\mathrm{Ti}$ and $\mathrm{TiO}_{2}$ nanoparticle arrays only exhibited broad spontaneous emission even at very high pumping energies approaching the bleaching condition for IR-140 dye $\left(0.8 \mathrm{~mJ} \mathrm{~cm}^{-2}\right)$ (Fig. 4a,b, Supplementary Fig. S11). Compared with Au nanoparticle arrays, the lasing emission wavelength in $\mathrm{Ag}$ nanoparticle arrays was shifted from $913 \mathrm{~nm}$ to $907 \mathrm{~nm}$, which is consistent with the blueshift of its resonant peak wavelength in 

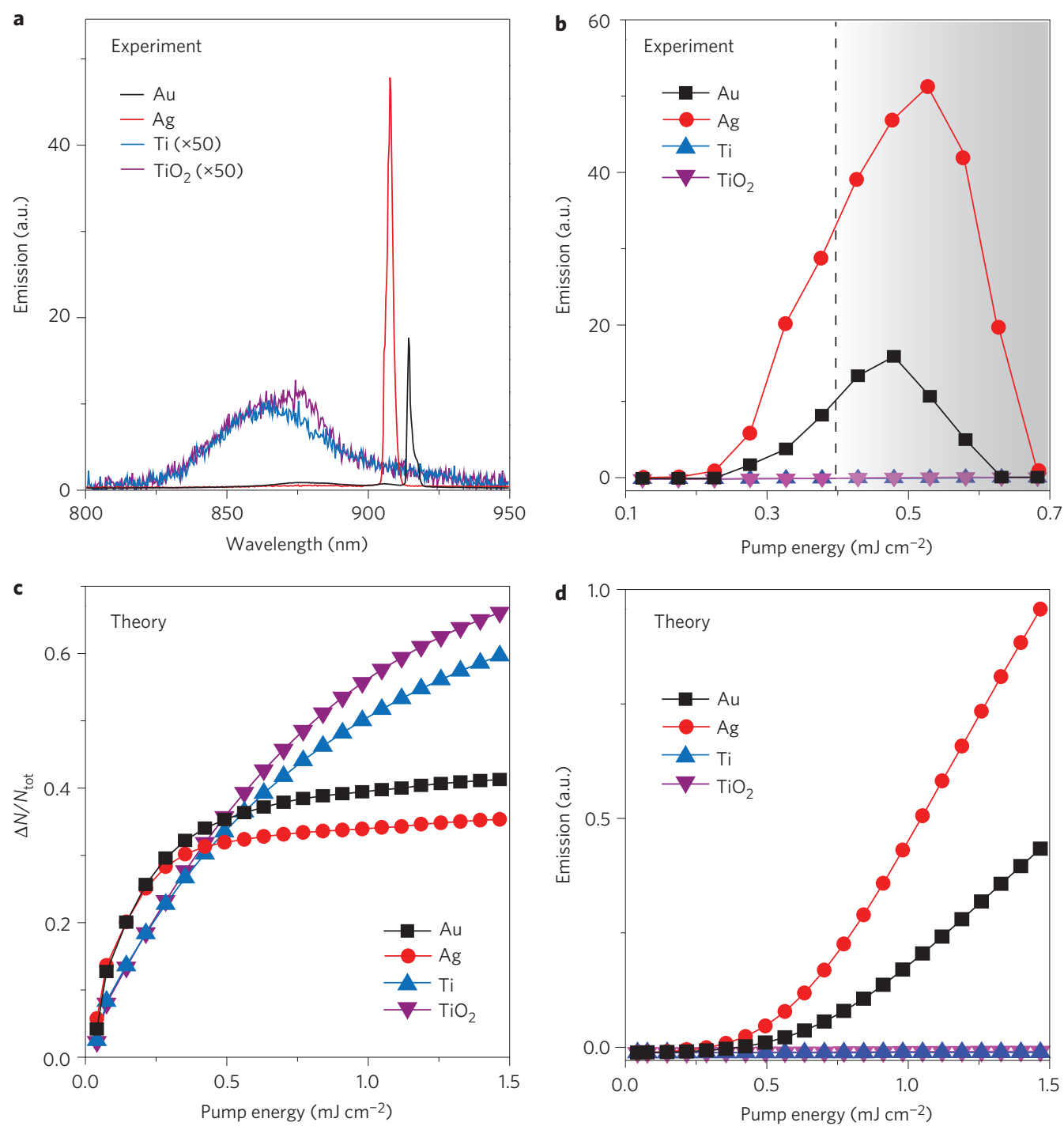

Figure 4 | Optical response of nanoparticle arrays surrounded by gain depends strongly on the materials of nanoparticles. a, Measured emission spectra of two-dimensional nanoparticle arrays of plasmonic and non-plasmonic materials $\left(\mathrm{Au}, \mathrm{Ag}, \mathrm{Ti}\right.$, TiO $\left.{ }_{2}\right)$ with gain media at a pump pulse energy of $0.43 \mathrm{~mJ} \mathrm{~cm}^{-2} \cdot \mathbf{b}$, Measured output emission intensity as a function of input pump pulse energy. The shaded region represents when off-angle amplified spontaneous emission emerges and surface-normal lasing emission becomes suppressed. c, Calculated average population inversion density $\Delta N / N_{\text {tot }}$ as a function of input pump pulse energy for gain close to nanoparticles (within $50 \mathrm{~nm}$ of the nanoparticle surface). $\mathbf{d}$, Calculated output emission intensity as a function of input pump pulse energy.

extinction measurements (Supplementary Fig. S10). In addition, Ag nanoparticle arrays exhibited smaller threshold pump energies and larger emission intensities compared with Au nanoparticle arrays under the same pump conditions because of smaller absorption losses over the entire visible to near-infrared range, as well as greater overlap between the plasmon resonance and dye emission wavelength. Calculations confirmed that only $\mathrm{Au}$ and Ag nanoparticle arrays could induce lasing emission with the population inversion clamped at threshold (Fig. 4c,d, Supplementary Fig. S9). In contrast, without a waveguide structure for vertical mode confinement, $\mathrm{Ti}$ and $\mathrm{TiO}_{2}$ nanoparticle arrays cannot provide any feedback for lasing emission, even if a high population inversion is built in the system (Fig. 4c).

In addition to facilitating strong dipole-dipole interactions, a high resonant dipole strength $|\mathbf{p}|$ in the plasmonic nanoparticles can also lead to a large $|E|^{2}$ and LDOS near the metal surface, which will significantly enhance the spontaneous emission rate. To verify the Purcell effect for emitters coupled to lattice plasmons, we measured the decay dynamics of excited IR-140 molecules in plasmonic and non-plasmonic nanoparticle arrays by femtosecond-transient absorption spectroscopy (see Methods and Supplementary Fig. S12). Without nanoparticle arrays, the transient absorption spectra of the gain reference as a function of pumpprobe delay was dominated by a broad peak at $575 \mathrm{~nm}$ because of excited-state absorption. The negative feature at $700-750 \mathrm{~nm}$ is due to ground-state bleaching (Supplementary Fig. S13). The mono-exponential decay time constant of the excited-state absorption was measured to be $936 \pm 72 \mathrm{ps}$, corresponding to the intrinsic total lifetime of IR-14027. For the IR-140 molecules in a uniform environment at room temperature, non-radiative decay dominates with a low quantum yield ${ }^{28}, \eta=\tau_{\mathrm{r} 0}^{-1} /\left(\tau_{\mathrm{r} 0}^{-1}+\tau_{\mathrm{nr}}^{-1}\right)=16 \%$, where $\tau_{\mathrm{r} 0}$ and $\tau_{\mathrm{nr}}$ are the intrinsic radiative and non-radiative decay times. In the presence of $\mathrm{Au}$ nanoparticle arrays, the transient absorption spectra yielded identical spectral features but with ultrafast dynamics (Fig. 5a).

The normalized transient kinetic traces monitored at $575 \mathrm{~nm}$ clearly show that $\mathrm{Ti}$ and $\mathrm{TiO}_{2}$ nanoparticle arrays did not induce fast decay kinetics for the excited states (Fig. 5b). In contrast, $\mathrm{Au}$ 

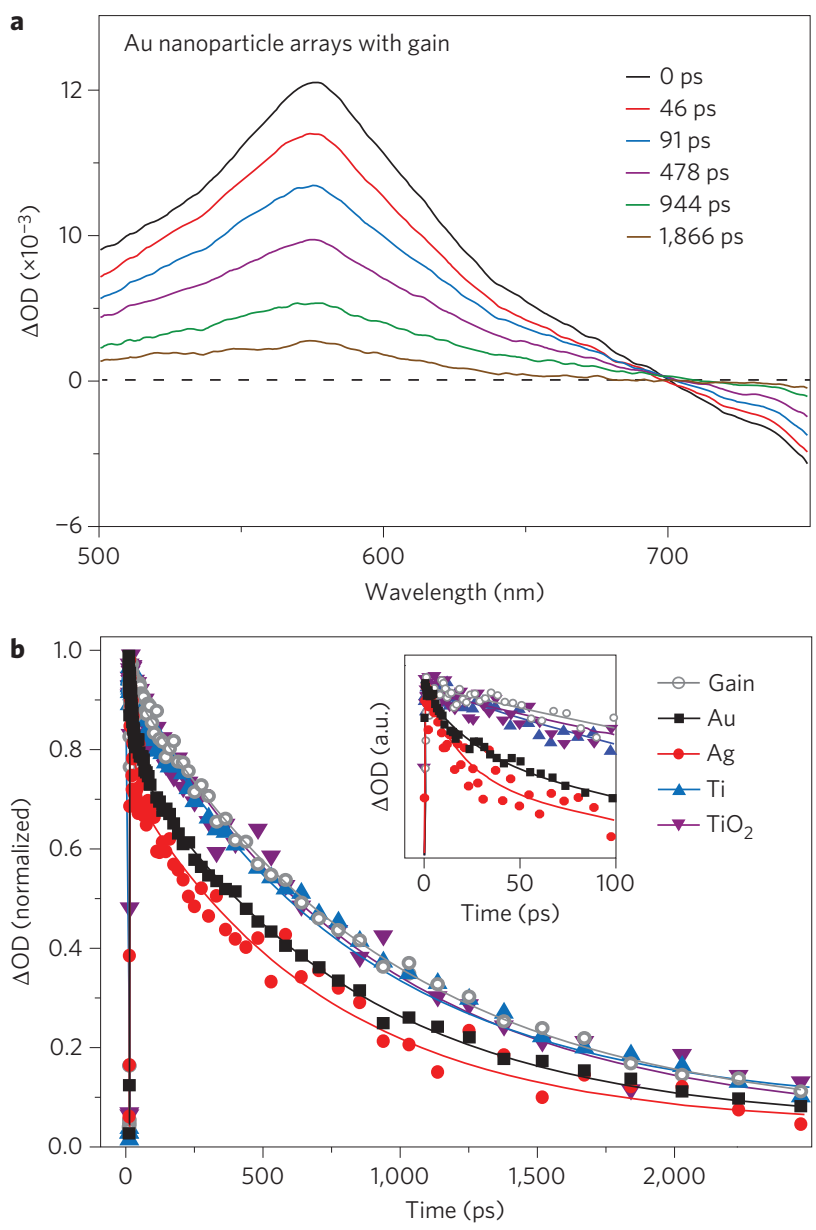

Figure 5 | Ultrafast decay rates of excited-state molecules indicate enhanced spontaneous emission rates by the Purcell effect. a, Transient absorption spectra of Au nanoparticle arrays surrounded by gain media (IR-140 dyes) at the indicated pump-probe delays. b, Measured change in optical density $\left(\Delta \mathrm{OD}=-\log _{10}\left(T_{\text {on }} / T_{\text {off }}\right)\right)$ as a function of pump-probe delay time for nanoparticle array samples of different materials.

and Ag nanoparticle arrays showed ultrafast depletion of the excited states, and thus the transient absorption kinetics needed to be fit to a sum of two exponential decay components

$$
I(t) \approx A_{1} \exp \left(-t / \tau_{\text {coupled }}\right)+A_{2} \exp \left(-t / \tau_{\text {uncoupled }}\right)
$$

The fast time constant $\tau_{\text {coupled }}$ was determined to be $28 \pm 6$ ps for Au nanoparticle arrays and $22 \pm 11$ ps for Ag nanoparticle arrays from excited-state molecules coupled to lattice plasmons with a Purcellenhanced spontaneous emission rate $\left(F^{\prime} / \tau_{\mathrm{r} 0}\right)$, where $F^{\prime}$ is the experimentally determined enhancement factor. The slow time constant $\tau_{\text {uncoupled }}$ was found to be $923 \pm 44$ ps for Au nanoparticle arrays and $879 \pm 131$ ps for Ag nanoparticle arrays, from excited-state molecules that did not interact with lattice plasmons; this is similar to the intrinsic lifetime of IR-140. We estimated $F^{\prime}$ to be 200 for Au nanoparticle arrays and 250 for Ag nanoparticle arrays using the expression

$$
\frac{1}{\tau_{\text {uncoupled }}}=\frac{1}{\tau_{\mathrm{r} 0}}+\frac{1}{\tau_{\mathrm{nr}}} \quad \text { and } \quad \frac{1}{\tau_{\text {coupled }}}=\frac{F^{\prime}}{\tau_{\mathrm{r} 0}}+\frac{1}{\tau_{\mathrm{r} 0}}+\frac{1}{\tau_{\mathrm{nr}}}
$$

$F^{\prime}$ of the spontaneous emission rate represents an average kinetic response of a large ensemble of dye emitters close to the plasmonic nanoparticles. The estimated $F^{\prime}$ from measurements matched well with our simulation results obtained by finite-difference time- domain (FDTD) analysis of a dipole emitter in the subwavelength vicinity of the nanoparticles (Supplementary Fig. S14). Thus, bandedge lattice plasmons supported in plasmonic nanocavity arrays can behave as lasing feedback with increased modulation speeds, because stimulated emission processes will be enhanced by the Purcell effect ${ }^{5,12}$.

In summary, we have described lasing action in strongly coupled plasmonic nanocavity arrays based on optical amplification of bandedge lattice plasmons with a near-zero group velocity. This phenomenon was used to create a new type of surface-emitting laser that combines the advantages of photonic band-edge lasers (for example, directional beam emission) with those of plasmonic nanolasers (for example, nanoscale optical confinement, ultrafast response). The generation of nanolocalized optical fields over large-area $\left(\mathrm{cm}^{2}\right)$ arrays of nanoparticles could enable a massively parallel enhancement of weak physical-chemical processes on the nanoscale, such as fluorescence and Raman scattering, photocatalysis and other nonlinear optical phenomena.

Note added in proof: After this Letter was accepted for publication, an analogous lasing system was reported ${ }^{31}$.

\section{Methods}

Sample fabrication. Au nanohole arrays fabricated by PEEL, a nanofabrication procedure combining photolithography, etching, electron-beam deposition and lift-off ${ }^{29}$, were used as a physical deposition mask to create a periodic array of nanoparticles of different materials $\left(\mathrm{Au}, \mathrm{Ag}, \mathrm{Ti}\right.$ and $\mathrm{TiO}_{2}$ ) over $\mathrm{cm}^{2}$ areas on glass substrates. All nanoparticle diameters $d$ were $130 \mathrm{~nm}$, the period $a_{0}$ of the square lattice was $600 \mathrm{~nm}$, and the height $h$ of the nanoparticles was $60 \mathrm{~nm}$. A thermally cured layer of polyurethane (NOA $83 \mathrm{H}, n=1.55$, Norland Product) mixed with infrared fluorescence dye molecules (IR-140, Sigma-Aldrich) was used as the gain medium. The concentration of IR-140 in the polyurethane layer was $2 \times 10^{18} \mathrm{~cm}^{-3}$, and the measured absorption and emission peaks of IR-140 in the polyurethane layer were $820 \mathrm{~nm}$ and $870 \mathrm{~nm}$. IR-140-polyurethane liquid $(1 \mu \mathrm{l})$ was dropped onto two-dimensional nanoparticle arrays on the glass substrate and then sandwiched between this and another glass substrate to avoid oxidation of the dye. The IR-140-polyurethane layer was then thermally cured on a hotplate at $110^{\circ} \mathrm{C}$ for $60 \mathrm{~s}$

Lasing measurements. A mode-locked Ti:sapphire laser with a regenerative amplifier ( $800 \mathrm{~nm}$ wavelength, $1 \mathrm{kHz}$ repetition rate, $40 \mathrm{fs}$ pulse width) was used to optically pump the nanoparticle samples at room temperature. The pump beam was focused to a 5-mm-diameter spot on the sample at an incident angle of $45^{\circ}$. The emission spectra were collected (detector collection angle $<10^{\circ}$ ) at different detection angles $\alpha$ and then coupled into a bundled optical fibre connected to a compact spectrometer (USB 2000, Ocean Optics, $0.3 \mathrm{~nm}$ resolution). The incident plane of the pump beam was aligned along the high-symmetry axis of the square lattice. The spatial pattern and divergence angle of the lasing beam were analysed using a high-resolution CCD beam profiler (LBR-HR, Newport, 1.4 Megapixel) placed at a distance of $15 \mathrm{~cm}$, normal to the sample surface.

Numerical simulations of active optical responses for nanoparticle arrays with gain. The active gain medium (dye molecules) was modelled as a four-level quantum system, and the dynamics of the energy-level populations were determined by solving rate equations coupled to electromagnetic fields obtained using an in-house three-dimensional FDTD code ${ }^{25,30}$. Simulations were carried out under periodic boundary conditions with zero in-plane wavevector, which is a reasonable simplification that can capture the main physics of band-edge lattice plasmon lasing at relatively small pump intensities (that is, when the contributions of off-angle spontaneous emission are small).

Transient absorption spectroscopy. Time-resolved transient absorption spectra were obtained as the change in optical density, $\Delta \mathrm{OD}=-\log _{10}\left(T_{\text {on }} / T_{\text {off }}\right)$, at different pump-probe delay times (250 fs resolution), where $T_{\text {on }}$ and $T_{\text {off }}$ are the

transmittance of the probe pulse with and without the pump beam. The $800 \mathrm{~nm}$, $40 \mathrm{fs}$ pulses out of the regenerative amplifier were split and used as the pump and probe beams. The pump beam $(200 \mathrm{~nJ} /$ pulse $)$ at $800 \mathrm{~nm}$ was directed to a delay line and subsequently focused into the sample with a diameter of $1.5 \mathrm{~mm}$. The whitelight-continuum probe pulse was generated by focusing the $800 \mathrm{~nm}$ beam into a 2.5-mm-thick sapphire disk. The white light was then focused into the sample (beam diameter, $0.7 \mathrm{~mm}$ ). The spectra of the transmitted continuum probe beam were recorded using a CCD-spectrometer (PIXIS 100B, Princeton Instruments; TRIAX 180 , HORIBA Scientific). The polarization of the continuum probe pulses was set to a magic angle $\left(54.7^{\circ}\right)$ to avoid polarization effects in the transient signal.

Received 9 March 2013; accepted 26 April 2013;

published online 16 June 2013 


\section{References}

1. Kogelnik, H. \& Shank, C. V. Coupled-wave theory of distributed feedback lasers. J. Appl. Phys. 43, 2327-2335 (1972).

2. Meier, M. et al. Laser action from two-dimensional distributed feedback in photonic crystals. Appl. Phys. Lett. 74, 7-9 (1999).

3. Noda, S., Yokoyama, M., Imada, M., Chutinan, A. \& Mochizuki, M. Polarization mode control of two-dimensional photonic crystal laser by unit cell structure design. Science 293, 1123-1125 (2001).

4. Matsubara, H. et al. GaN photonic-crystal surface-emitting laser at blue-violet wavelengths. Science 319, 445-447 (2008)

5. Altug, H., Englund, D. \& Vuckovic, J. Ultrafast photonic crystal nanocavity laser. Nature Phys. 2, 484-488 (2006).

6. Englund, D., Atlug, H., Ellis, B. \& Vuckovic, J. Ultrafast photonic crystal lasers. Laser Photon. Rev. 2, 264-274 (2008).

7. Noginov, M. A. et al. Demonstration of a spaser-based nanolaser. Nature 460 1110-1168 (2009).

8. Oulton, R. F. et al. Plasmon lasers at deep subwavelength scale. Nature 461, 629-632 (2009).

9. Stockman, M. I. The spaser as a nanoscale quantum generator and ultrafast amplifier. J. Opt. 12, 024004 (2010).

10. Novotny, L. \& Hecht, B. Principles of Nano-Optics (Cambridge Univ. Press, 2006).

11. Purcell, E. M. Spontaneous emission probabilities at radio frequencies. Phys. Rev. 69, 681 (1946).

12. Ma, R-M., Oulton, R. F., Sorger, V. J. \& Zhang, X. Plasmon lasers: coherent light source at molecular scales. Laser Photon. Rev. 7, 1-21 (2012).

13. Maier, S. A. Plasmonics: Fundamentals and Applications (Springer, 2007).

14. Zou, S. L., Janel, N. \& Schatz, G. C. Silver nanoparticle array structures that produce remarkably narrow plasmon lineshapes. J. Chem. Phys. 120, 10871-10875 (2004).

15. Auguie, B. \& Barnes, W. L. Collective resonances in gold nanoparticle arrays. Phys. Rev. Lett. 101, 143902 (2008).

16. Chu, Y. Z., Schonbrun, E., Yang, T. \& Crozier, K. B. Experimental observation of narrow surface plasmon resonances in gold nanoparticle arrays. Appl. Phys. Lett. 93, 181108 (2008).

17. Kravets, V. G., Schedin, F. \& Grigorenko, A. N. Extremely narrow plasmon resonances based on diffraction coupling of localized plasmons in arrays of metallic nanoparticles. Phys. Rev. Lett. 101, 087403 (2008).

18. Vecchi, G., Giannini, V. \& Rivas, J. G. Surface modes in plasmonic crystals induced by diffractive coupling of nanoantennas. Phys. Rev. B 80, 201401 (2009).

19. Zhou, W. \& Odom, T. W. Tunable subradiant lattice plasmons by out-of-plane dipolar interactions. Nature Nanotech. 6, 423-427 (2011).

20. Zhou, W., Hua, Y., Huntington, M. D. \& Odom, T. W. Delocalized lattice plasmon resonances show dispersive quality factors. J. Phys. Chem. Lett. 3, 1381-1385 (2012).

21. Loncar, M. et al. Experimental and theoretical confirmation of Bloch-mode light propagation in planar photonic crystal waveguides. Appl. Phys. Lett. 80, 1689-1691 (2002).
22. Baba, T. Slow light in photonic crystals. Nature Photon. 2, 465-473 (2008)

23. Vecchi, G., Giannini, V. \& Rivas, J. G. Shaping the fluorescent emission by lattice resonances in plasmonic crystals of nanoantennas. Phys. Rev. Lett. 102, 146807 (2009)

24. Samuel, I. D. W., Namdas, E. B. \& Turnbull, G. A. How to recognize lasing. Nature Photon. 3, 546-549 (2009).

25. Siegman, A. E. Lasers (University Science Books, 1986).

26. Samuel, I. D. W. \& Turnbull, G. A. Organic semiconductor lasers. Chem. Rev. 107, 1272-1295 (2007).

27. Wang, D. L. et al. Investigation on photoexcited dynamics of IR-140 dye in ethanol by femtosecond supercontinuum-probing technique. J. Opt. A 4, 155-159 (2002)

28. Rurack, K. \& Spieles, M. Fluorescence quantum yields of a series of red and nearinfrared dyes emitting at 600-1000 nm. Anal. Chem. 83, 1232-1242 (2011).

29. Henzie, J., Lee, M. H. \& Odom, T. W. Multiscale patterning of plasmonic metamaterials. Nature Nanotech. 2, 549-554 (2007)

30. Nagra, A. S. \& York, R. A. FDTD analysis of wave propagation in nonlinear absorbing and gain media. IEEE Trans Antennas Propag. 46, 334-340 (1998).

31. Van Beijnum, F. et al. Surface plasmon lasing observed in metal hole arrays. Phys. Rev. Lett. 110, 206802 (2013).

\section{Acknowledgements}

This work was supported by the NSF-MRSEC program at the Materials Research Science and Engineering Center at Northwestern University (DMR-1121262; W.Z., J.Y.S., M.D., G.C.S., T.W.O.), the Initiative for Sustainability and Energy at Northwestern (ISEN) Faculty Booster Award (J.Y.S., C.H.K., D.T.C.) and the US Department of Energy (DOE), Office of Science, Office of Basic Energy Sciences (DE-SC0004752; M.D., G.C.S.). Transient absorption measurement and data analysis were supported as part of the ANSER Center, an Energy Frontier Research Center funded by the DOE (DE-SC0001059; D.T.C., M.R.W.).

\section{Author contributions}

W.Z. and T.W.O. conceived the idea of a new surface-emitting laser based on strongly coupled plasmonic nanocavity arrays. W.Z. fabricated the devices, carried out the angleresolved optical measurement, and performed FDTD numerical simulations of the passive optical responses of the devices. M.D. developed the numerical methods to simulate the active optical responses of the device. J.Y.S., C.H.K. and W.Z. carried out lasing and transient absorption measurements, and C.H.K. and D.T.C. set up the transient absorption measurements. T.W.O., G.C.S., D.T.C. and M.R.W. guided the experimental and theoretical investigations. W.Z. and T.W.O. analysed the data and wrote the manuscript.

\section{Additional information}

Supplementary information is available in the online version of the paper. Reprints and permissions information is available online at www.nature.com/reprints. Correspondence and requests for materials should be addressed to T.W.O.

\section{Competing financial interests}

The authors declare no competing financial interests. 